\title{
Atypical Presentation of Gitelman Syndrome (GS) Masquerading as Hypokalaemic Periodic Paralysis
}

\author{
Akhilesh Annadatha ${ }^{1}$, Sourya Acharya ${ }^{2}$, Amol Andhale $^{3}$, Sree Karthik Pratapa ${ }^{3}$
}

${ }^{1}$ Department of Medicine, Datta Meghe Institute of Medical Sciences (Deemed to Be University), Sawangi Meghe, Wardha, Maharashtra, India. ${ }^{2}$ Department of Medicine, Datta Meghe Institute of Medical Sciences (Deemed to Be University), Sawangi Meghe, Wardha, Maharashtra, India. ${ }^{3}$ Department of Medicine, Datta Meghe Institute of Medical Sciences (Deemed to Be University), Sawangi Meghe, Wardha, Maharashtra, India. ${ }^{4}$ Department of Medicine, Datta

Meghe Institute of Medical Sciences (Deemed to Be University), Sawangi Meghe, Wardha, Maharashtra, India.

\section{INTRODUCTION}

Gitelman syndrome once considered to be a subset of Barter's syndrome, is a disorder of the distal tubule.1,2 It is an inherited disorder of the thiazide sensitive sodiumchloride transport channel. It is characterised by its main clinical features of metabolic alkalosis, hypomagnesemia, hypokalaemia and hypocalciuria. It's an infrequently diagnosed condition where there diagnosis is made based on the clinical and the biochemical parameters of the disease. We present a case of Gitelman syndrome in a young male who presented with recurrent episodes of weakness to different private hospitals with documented hypokalaemia and labelled as hypokalaemic periodic palsy (HPP), presented to us with quadriparesis and on investigation diagnosed as GS.

\section{PRESENTATION OF CASE}

A 23 year old male, daily wage labourer presented to us, with complaints of abdominal pain, three episodes of vomiting, and weakness in all four since 2 days. He had no history of fever, headache, convulsions, urinary incontinence, tingling sensation in limbs, diarrhoea, and constipation. He previously had three episodes of similar complaints and was treated as hypokalaemic paralysis.

On examination, he was afebrile, pulse was 86 per minute, regular, blood pressure was 110 / $70 \mathrm{~mm}$ hg, right arm supine position, normal heart sounds were heard, chest was clear, per abdomen was soft. CNS examination revealed: higher functions normal. Speech was normal. Cranial nerve examination was normal. Power in upper limbs: 3 / 5, and lower limbs 4 / 5 / deep tendon reflexes were diminished, planters were bilateral mute. Sensory examination was unremarkable.

Lab investigations : $\mathrm{Hb}-11.1 \mathrm{gm} \%$,WBC count - 9200 cells / cumm, platelet count - 1.15 lakhs / $\mathrm{mm}^{3}$, serum creatinine was $1.8 \mathrm{mg} \%$, urea - $47 \mathrm{mg} \%$, sodium - 124 $\mathrm{mEq} / \mathrm{L}$, potassium - $2.0 \mathrm{mEq} / \mathrm{L}$, magnesium - $1.2 \mathrm{mEq} / \mathrm{L}$. Total CPK was $522 \mathrm{U} / \mathrm{L}$ (normal; 26 - 192 U), urine myoglobin was positive suggesting rhabdomyolysis. USG Kidney-ureter-bladder was normal. Electrocardiogram on admission showed U waves. (Figure 1) Arterial blood gas analysis of the patient revealed a Bicarbonate level of $36 \mathrm{mEq} / \mathrm{L}, \mathrm{Ph}-7.52$ with normal $\mathrm{pO}_{2}$ and $\mathrm{pCO}_{2}$ (metabolic alkalosis). Urine was sent for electrolytes which revealed Hypocalciuria $12 \mathrm{mg} /$ day. Patient had hypokalaemic metabolic alkalosis, hypomagnesemia with hypocalciuria and was hence suspected as a case of Gitelman syndrome. He was started on oral supplements of magnesium and potassium and the levels have steadily increased and was subsequently normalised.
Corresponding Author: Dr. Sourya Acharya,

Professor,

Department of Medicine,

Datta Meghe Institute of Medical

Sciences (Deemed to Be University),

Sawangi, Meghe, Wardha,

Maharashtra, India.

E-mail: souryaacharya74@gmail.com

DOI: 10.14260/jemds/2020/623

How to Cite This Article:

Annadatha A, Acharya S, Andhale A, et al. Atypical presentation of gitelman syndrome (GS) masquerading as hypokalemic periodic paralysis. J Evolution Med Dent Sci 2020;9(38):2857-2858, DOI: 10.14260/jemds/2020/623

Submission 26-04-2020,

Peer Review 14-08-2020,

Acceptance 20-06-2020,

Published 21-09-2020.

Copyright (c) 2020 Akhilesh Annadatha et al. This is an open access article distributed under Creative Commons Attribution License [Attribution 4.0 International (CC BY 4.0)] 


\section{DISCUSSION}

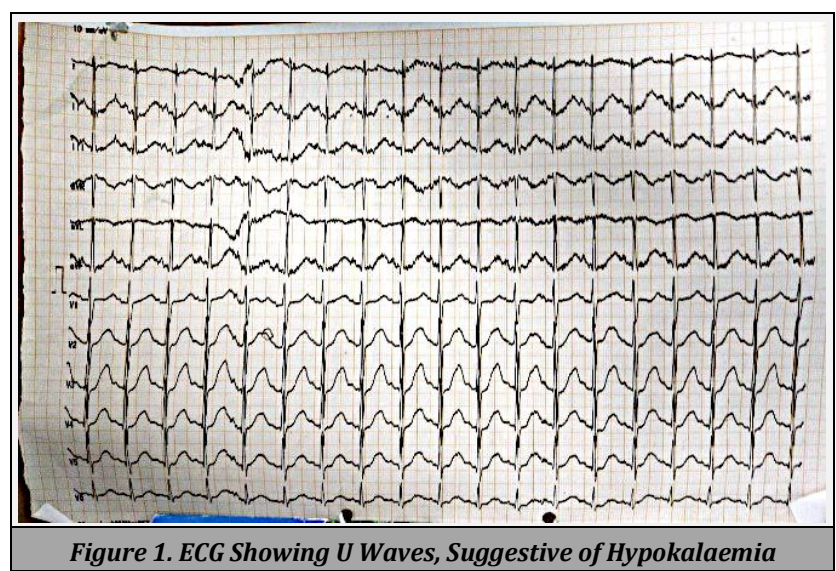

Gitelman's syndrome, an autosomal recessive disorder is characterised by metabolic abnormalities like hypokalaemic metabolic alkalosis, hyperreninemia, hyperplasia of the juxtaglomerular apparatus, hyper-aldosteronism and in some, hypomagnesemia. It occurs due to mutations in solute carrier family 12, member 3 gene, (SLC12A3), which encodes the thiazide sensitive $\mathrm{NaCl}$ cotransporter (NCC). ${ }^{3}$ The common differentials include Bartter's syndrome and primary hyperaldosteronism. $^{2}$ It is differentiated from primary hyperaldosteronism by norm tension.

Any patient with recurrent episodes of generalised weakness and having hypokalaemia, this is one diagnosis that should not be missed. This also emphasizes the importance of an arterial blood gas in coming to a diagnosis which would help in treating the condition. Gitelman syndrome is a diagnosis of exclusion when other conditions leading to hypokalaemia and hypomagnesemia are excluded. A complete metabolic panel is helpful in diagnosis. Aldosterone antagonists or Sodium channel blockers are used in cases where the hypokalaemia is not corrected by routine methods. ${ }^{4,5}$ The spectrum of symptoms can range from simple weakness to more severe symptoms like tetany, paralysis or rhabdomyolysis. Our patient had paralysis, rhabdomyolysis and acute kidney injury which are rarely seen in GS. Long term magnesium supplementation and diets rich in sodium and potassium are encouraged in these cases.

Financial or Other Competing Interests: None.

\section{REFERENCES}

[1] Seyberth HW, Schlingmann KP. Bartter- and Gitelman-like syndromes: salt-losing tubulopathies with loop or DCT defects. Pediatr Nephrol 2011;26(10):1789-802.

[2] Tsuchiya H, Kamoi K, Soda S, et al. Gitelman's syndrome first diagnosed as Bartter's syndrome. Intern Med 2001;40(10):1011-4.

[3] Knoers NV, Levtchenko EN. Gitelman syndrome. Orphanet J Rare Dis 2008;3:22.

[4] Lin SH, Cheng NL, Hsu YJ, et al. Intrafamilial phenotype variability in patients with Gitelman syndrome having the same mutations in their thiazide-sensitive sodium/chloride cotransporter. Am J Kidney Dis 2004;43(2):304-12.

[5] Cruz DN, Shaer AJ, Bia MJ, et al. Gitelman's syndrome revisited: an evaluation of symptoms and health-related quality of life. Kidney Int 2001;59(2):710-7. 